\title{
Abdomen Actin' Up: A Unique Presentation of Disseminated Abdominal Actinomycosis
}

\author{
Arpana Paruchuri, MD, Brianna J. Shinn, MD, and Rino Sato, MD
}

\section{INTRODUCTION}

Abdominal actinomycosis is a chronic, indolent disease characterized by nonspecific symptoms such as fatigue, weight loss, fever, and abdominal pain. Actinomyces is a genus of fastidious, gram-positive, non-acid-fast, branching filamentous bacilli characterized by sulfur granules that is normally found in oral flora and inhabits the gastrointestinal (Gl) tract. ${ }^{1}$ Actinomyces infections are relatively rare, however when present, they have the ability to invade multiple organs and disseminate throughout multiple body cavities. ${ }^{2}$ Factors that increase the risk of developing actinomycosis include poor oral hygiene, alcoholism, and preexisting dental disease. Intrauterine devices (IUDs) also increase the risk of developing pelvic actinomycosis. ${ }^{3}$ Over the past $10-20$ years, actinomycosis is being diagnosed with increasing frequency and should be considered in the differential for patients presenting with indolent abdominal symptoms along with risk factors. ${ }^{4}$

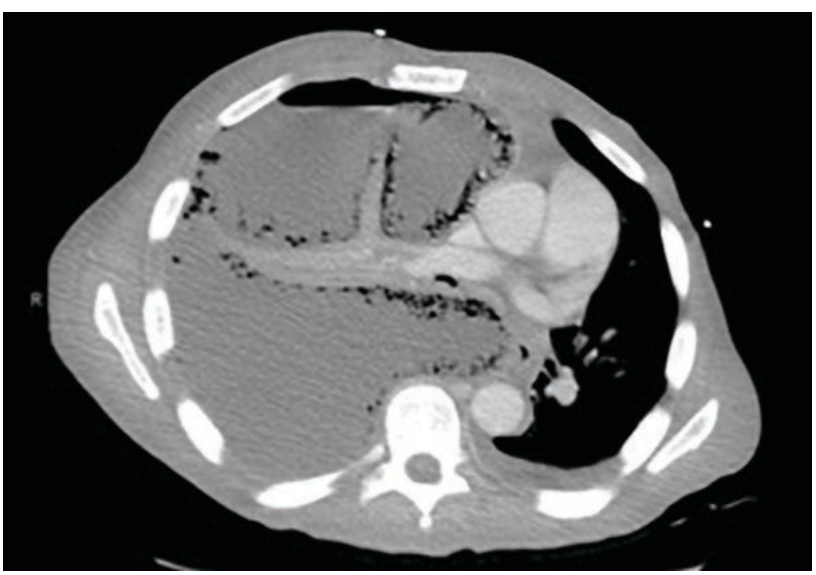

Figure 1. CT Chest demonstrating right-sided pleural effusion with complete collapse of the right lung

\section{CASE PRESENTATION}

A 57-year old male with a history of untreated hepatitis C, treated tuberculosis (TB), and recent incarceration presented to the emergency room with abdominal pain, shortness of breath and weight loss. Of note, he had undergone a liver biopsy two weeks prior to presentation to further evaluate a liver lesion that showed acute and chronic inflammation without evidence of malignancy or infection.

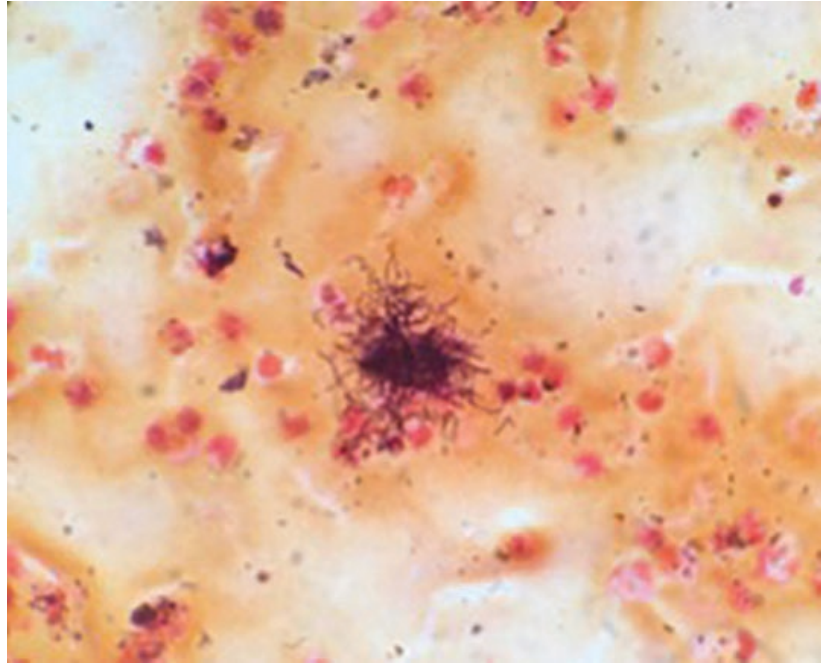

Figure 2. Actinomyces meyeri from the patient's abdominal wall abscess.

On exam, he was afebrile, had a blood pressure of $85 / 70$ $\mathrm{mmHg}$, and an oxygen saturation of $84 \%$ on room air. The patient had poor dentition, decreased breath sounds bilaterally, and multiple abdominal masses palpable in the left lower quadrant and along the liver border. Labs were remarkable for an elevated lactate of $2.3 \mathrm{mmol} / \mathrm{L}$, hemoglobin of $7.6 \mathrm{~g} / \mathrm{dL}$, and albumin level of $1.9 \mathrm{~g} / \mathrm{dL}$. His white blood cell (WBC) count and arterial blood gas were within normal limits. Imaging revealed a large right-sided pleural effusion with septations and complete collapse of the right lung (Figure 1), as well as multiple intra-abdominal abscesses with air-fluid levels.

\section{DIFFERENTIAL DIAGNOSIS}

Due to his history of previously treated TB, recent incarceration, and recent liver biopsy, the source of infection was thought to be due to TB or a bacterial source introduced from his recent liver biopsy. Given the imaging findings which demonstrated a pleural effusion with septations, the patient had a surgical chest tube placed which drained over three liters of purulent fluid. Based on Light's criteria the pleural fluid was classified as an exudative effusion, with 16,075 WBCs $/ \mathrm{mm} 3$, Glucose $<2.0 \mathrm{mg} / \mathrm{dL}$, LDH of $4110 \mathrm{IU} / \mathrm{L}$, and a $\mathrm{pH}$ of 7.0 . Based on these results and the presence of pus in the pleural space, he was diagnosed with an empyema. 


\section{OUTCOME AND FOLLOW-UP}

After the patient's surgical chest tube was placed, he underwent drainage of the abdominal wall abscesses. Cultures from both the pleural fluid and abdominal abscess fluid grew Actinomyces meyeri and the patient was diagnosed with disseminated actinomycosis (Figure 2). The patient ultimately required a video-assisted thoracoscopic surgery (VATS) and decortication during which time he was noted to have a fistulous connection between a hepatic abscess and the right hemithorax. Since the patient's poor dentition was presumed to be the initial source of this infection, he also underwent tooth extraction. He was treated with intravenous Unasyn for six months without transition to oral Penicillin given his complicated presentation. Unasyn was chosen due on its broader coverage and its ability to treat any concomitant infections in this complicated patient. Repeat imaging of his abdomen 2 months after his presentation showed a decrease in size of his intra-abdominal collections.

\section{DISCUSSION}

Abdominal actinomycosis is an infection in the abdomen most commonly caused by Actinomyces israelii, which is normally an inhabitant of the oral cavity and Gl tract. ${ }^{1}$ Actinomyces meyeri, while a less common cause of abdominal actinomycosis, has a greater propensity for causing disseminated disease. ${ }^{3}$ Although Actinomyces normally has low virulence, it acquires its pathogenicity through invasion of necrotic tissue or local infections or trauma. As the infection progresses, granulomatous tissue, extensive reactive fibrosis and necrosis, abscesses, draining sinuses, and fistulas can be seen. The most frequent sites of infection include the cervicofacial region (50\% of cases), abdominal cavity (20\%), and thoracic cavity (15-20\%). ${ }^{2}$ Patients with abdominal actinomycosis, including our patient, generally present with a chronic, indolent course of nonspecific symptoms such as fatigue, fever, weight loss, and abdominal pain. Physical exam findings may include a palpable abdominal mass, visible sinus tracts, or fistulas and labs can demonstrate anemia and leukocytosis. ${ }^{5}$ Culturing abscess fluid is the most effective way to diagnosis an Actinomyces infection. Once diagnosed, the treatment of choice is IV Penicillin G for 4-6 weeks followed by Amoxicillin for 6-12 months for large abdominal lesions or draining sinus tracts. ${ }^{2}$

Abdominal actinomycosis is a difficult condition to diagnose due to its rarity, nonspecific symptoms, and its resemblance of more common conditions such as malignancy, Crohn's disease, and tuberculosis. ${ }^{2}$ Our patient's case was further complicated by the fact that the infection invaded the thoracic cavity and led to development of an empyema and disseminated actinomycosis. In our patient's case, this invasion of the pleural space was thought to be due to local trauma and direct seeding from the liver biopsy. However, there are cases reports in the literature that describe fistula development between different body cavities allowing for disseminated disease. ${ }^{3}$ While fistula development is rare, it is an important complication to be aware of since disseminated disease can be difficult to control and treat.

While abdominal actinomycosis is a relatively rare condition, this case is significant given the rising incidence of this disease over the past 10-20 years. It is important for physicians to be educated about this disease especially given its vague and non-specific symptoms so that it can be included in the differential diagnoses for patients presenting with an insidious onset of abdominal symptoms as well as predisposing risk factors.

\section{KEY POINTS}

- While still rare, the incidence of abdominal actinomycosis is increasing

- Abdominal actinomycosis presents with a chronic, indolent course of nonspecific symptoms similar to other, more common conditions and, should be considered in patients with indolent abdominal symptoms and risk factors including poor dental hygiene, dental disease, and alcoholism.

- The diagnosis is based on abscess fluid culture growing Actinomyces.

- Treatment of actinomycosis is generally with a long course of IV Penicillin G later transitioned to oral Amoxicillin.

\section{REFERENCES}

1. Wagenlehner FME, Mohren B, Naber KG, Männl HFK. Abdominal actinomycosis. Clinical Microbiology and Infection. 2003:9:881-885.

2. Wong VK, Turmezei TD, Weston VC. Actinomycosis. BMJ. 2011;343.

3. Valour F, Senechal A, Dupieux C, et al. Actinomycosis: etiology, clinical features, diagnosis, treatment, and management. Infection and Drug Resistance. 2014;7:183-197.

4. Cintron JR, Pino AD, Duarte B, Wood D. Abdominal actinomycosis: Report of two cases and review of the literature. Diseases of the Colon \& Rectum. 1996;39(1): 105-108.

5. Filipović B, Milinić N, Nikolić G, Ranthelović T. Primary actinomycosis of the anterior abdominal wall: case report and review of the literature. Journal of Gastroenterology and Hepatology. 2005:20:517-520. 